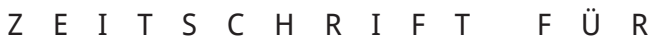

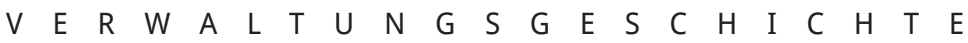

B A N D 1,20016

S E I T E $3-9$

D O I : $10.2478 / \mathrm{ADH}-2018-0001$

\title{
Verwaltungsgeschichte im Dialog
}

Einleitung

PETER BECKER, STEFAN NELLEN

In den Blick zu nehmen waren also nicht nur

Behörden und Beamte in Staat und Stadt, nicht nur

Wirtschaft und Wissenschaft, nicht nur Recht

und Religion oder Macht und Moral - auch Kunst,

Architektur, Literatur, Natur, Philosophie und

Technik in ihrem Bezug zum Verwaltungshandeln. ${ }^{1}$

Mit diesen Worten charakterisiert Erk Volkmar Heyen in seinem einleitenden Beitrag über die Entstehung und Arbeitsweise des Jahrbuchs für europäische Verwaltungsgeschichte rückblickend und nach wie vor treffend die Herausforderungen an eine transdisziplinäre Auseinandersetzung mit Staat und Verwaltung aus historischer Perspektive. Damit ist nicht nur der Austausch zwischen mehreren wissenschaftlichen Disziplinen angesprochen, sondern ebenso die Kommunikation mit Praktikern der Verwaltung - eine Auseinandersetzung, die für Historikerinnen und Historiker oft genug nur vermittelt über schriftliche Zeugnisse möglich ist. Eine so verstandene Verwaltungsgeschichte ist in einem Dialograum situiert, den wir vor allem mit den Instrumenten einer kulturwissenschaftlichen Diskurs- und Praxisforschung öffnen wollen.

Die Gründung einer neuen Plattform für die Beschäftigung mit der Geschichte von Staat und Verwaltung ist aus unserer Sicht dadurch gerechtfertigt, dass sich zwar im englischsprachigen Raum eine Reihe von hervorragenden Zeitschriften mit Verwaltung aus sozialwissenschaftlicher, oft normativer Perspektive auseinandersetzt, aber keine einen interdisziplinären, transregionalen und transepochalen Diskurs über Staat und Verwaltung pflegt. Genau darum geht es Administory²: Verwaltung zu historisieren und zwar aus disziplinär, regional und zeitlich übergreifenden Perspektiven. Um diese Vielfalt nicht zur Beliebigkeit werden zu lassen und die Anschlussfähigkeit der Beiträge an Debatten über Staat, Gesellschaft und Politik sicherzustellen, haben die einzelnen Bände der Zeitschrift jeweils einen Themenschwerpunkt. Außerhalb dieses Schwerpunkts bietet jeder Band auch die Möglichkeit, methodische Innovationen, Kommentare zu aktuellen Fragen der Verwaltungs- 
geschichte oder Forschungsberichte zu veröffentlichen. Zudem enthält jeder Band die Relektüre eines klassischen Textes zur Verwaltungsgeschichte, die seine Aktualität für verwaltungshistorische Fragestellungen auslotet. Wie der zweisprachige Titel und Untertitel der Zeitschrift anzeigt, erscheinen die Beiträge sowohl in deutscher wie auch in englischer Sprache.

Verwaltung tangiert nicht nur vielfältige Belange des Zusammenlebens von Menschen und Dingen, sondern kreuzt und vermengt auch wissenschaftliche Zugriffe auf sie. Anthropologie, Geschichtswissenschaften, Medienwissenschaften, Literaturwissenschaften, Philosophie, Politikwissenschaften, Rechtswissenschaften, Soziologie, Verwaltungswissenschaften, Wirtschaftswissenschaften, Wissenschaftsgeschichte und viele weitere Disziplinen beschäftigen sich mit divergierenden Erkenntnisinteressen und aus unterschiedlichen Warten mit (der) Verwaltung. Dieser programmatische Standpunkt hat uns dazu angeregt, im ersten Band die Dialogsituation zu reflektieren, wie sie auch in der Fotocollage auf dem Titel zum Ausdruck kommt. Wir wollten nicht eine explizite Theoriedebatte lancieren, weil dadurch das Fremde häufig durch hoch reflektierte Argumente vollständig inkorporiert wird. Wir haben vielmehr Autoren mit unterschiedlichem disziplinärem und theoretischem Hintergrund dazu eingeladen, sich ausgehend von ihren eigenen Forschungsarbeiten in einen Dialog mit der Verwaltungsgeschichte zu begeben. Wir möchten an dieser Stelle unseren Dank an alle Kolleginnen und Kollegen zum Ausdruck bringen, dass sie sich auf diese Herausforderung eingelassen und die Produktion eines spannenden Bandes mit unterschiedlichen Perspektiven auf Staat und Verwaltung ermöglicht haben.

Das Titelbild präsentiert eine Art Allegorie dieser Verwaltungsgeschichte im Dialog. Die Fotocollage, die wir eigens für diesen Band haben anfertigen lassen, zeigt auf der linken Seite den Körper eines Historikers, der einen zusammengerollten Plan und ein DIN-A4-Blatt in seiner rechten Hand hält. Auf seinen Hals montiert ist der Kopf von Niklas Luhmann, dem Verwaltungsbeamten unter den Soziologen und aktuellen theoretischen Kopf der Verwaltung. Luhmanns Kopf blickt mit offenem Mund nach links hoch zu einer anderen hybriden Gestalt im Anzug - halb Vogel, halb Mensch. Der Hühnermann steht seitlich zum Luhmann-Historiker und blickt seinerseits mit großen Augen streng auf ihn herab. Alles situiert in einem artifiziellen, verwischten Ambiente. In dieser Komposition haben wir das Setting der erwähnten Dialogsituation gezielt szugerichtet،. Links der Verwaltungshistoriker, der - verwundert, befremdet - mit seinem Gegenstand - einem Beamten beziehungsweise der Verwaltung - kommuniziert. Der Hühnermann kann aber umgekehrt auch eine fremde Disziplin verkörpern, die gebannt die Verwaltungsgeschichte in den Blick nimmt. Die beiden Figuren sind dann nicht nur einander, sondern als Hybride auch sich selbst fremd, insofern sie auch ein anderer sind; zugleich sind sie einander nicht nur fremd, sondern als Hybride auch verwandt.

Unsere künstlerische Strategie sehen wir durchaus in Analogie zu einer wissenschaftlichen Reflexion über Staat und Verwaltung. Diese schafft sich durch die Wahl der konzeptuellen Grundlagen und der methodischen Instrumente einen Projektraum, der in einem ähnlich spannungsreichen Bezug zu 
dem untersuchten Wirklichkeitsbereich steht, wie der Begegnungsraum, in dem der Kopf Luhmanns auf den Hühnermann trifft. Dieser steht für einen transdisziplinären Austausch, der mit Aneignungen und Verfremdungen operiert, um das konzeptuell andere in die eigene Forschungswelt zu integrieren. Diesen Prozess sehen wir durch diese Figur von David Altmejd auf eine hintersinnige Weise, als Aneignung des Fremden im Eignen, veranschaulicht. ${ }^{3}$ Dem Sakkoträger Luhmann steht eine ebenfalls im Jackett erscheinende Kunstfigur gegenüber, die aufgrund des Aussehens nur teilweise in der Welt eines Luhmann verankert ist. Das scheint uns eine treffende Darstellung unserer eigenen Bemühungen, mit der Fremdheit unserer Forschungsgegenstände und interdisziplinären theoretischen Anregungen zurechtzukommen.

Um dem Facettenreichtum unseres Forschungsgegenstands zu entsprechen, beziehen wir uns auf einen möglichst - auch zeitlich - offenen Begriff von Verwaltung, wie ihn etwa Cornelia Vismann in ihren Akten-Studien herausgearbeitet hat. In der "Kleine[n] Grammatologie für Akten «, der Einleitung zu ihrem Akten-Buch, konfrontiert Vismann den Verwaltungsforscher mit einer Fremdheitserfahrung, indem sie den zweiten Teil von Claude Lévi-Strauss' berühmter Schreibstunde bei den Nambikwara neu interpretiert. Sie deutet die Szene, in welcher der Häuptling mittels eines Papiers mit verschnörkelten Linien Geschenke verteilt, als einen Verwaltungsakt: Der Häuptling verwendet das Papier als Liste. Hier lässt sich eine Logik erkennen, wie wir sie im Blick auf Luhmann und den Hühnermann beschrieben haben. Mit einem Unterschied: Die Koexistenz fremder Gestalten wird hier für einen Praxisraum behauptet. Der Anzugträger im österreichischen Ministerium, der elektronische Listen bearbeitet, sieht sich plötzlich nicht nur in die Traditionslinie eines honorigen Mitglieds der habsburgischen Zentralverwaltung gesetzt - neben ihm nimmt nun auch der Häuptling der Nambikwara Platz.

Mit einer solchen Strategie lassen sich plausible Genealogien erstellen. Bezugnehmend auf Jack Goody beschreibt Vismann das Anfertigen von Listen "als Grundoperation jeder Verwaltung «: ${ }^{4}$ "Listen programmieren die Entstehung von Akten: vorgeschriebene Schrittfolgen, als Liste anschreibbare und abzuarbeitende Geschäftsanweisungen. Das heißt: Operative Zeichen [wie "acc, epi, exp, edivi oder $f$ " am Rand von Akten] steuern eine Akte konsekutiv vom ersten Akt, dem Anlegen, bis zu ihrer Ablage. [...] Es sind genauer gesagt diese nicht-syntaktischen Zeichen oder Operatoren, die Akten ohne weitere Kommunikation in die Umlaufbahnen der Verwaltungen schicken, immer begleitet von der bürokratischen Erlösungsformel z.d.A. "Aber "Listen programmieren den Lauf der Akten nicht nur, sondern dokumentieren ihn uno actu auch, wenn sie den Vollzug von Amtshandlungen vermerken. ${ }^{5}$

Ein solcher Zugang bringt eine kulturwissenschaftliche Perspektive in den Dialograum der Verwaltungsgeschichte. Wenn Listen als »Grundoperation jeder Verwaltung “ taxiert werden, dann rückt Verwaltung als Gebrauch von operativen Zeichen zur Steuerung von Vorgängen beziehungsweise zur "Verkettung der Operationen selbst« in den Blick, wie Erhard Schüttpelz argumentiert. Damit sieht man sich unvermittelt in den Denkraum der AkteurNetzwerke-Theorie von Bruno Latour versetzt, in der auch nicht menschliche 
Akteure wie Verwaltungsakten eine eigene Handlungsmächtigkeit erhalten. ${ }^{6}$ Verwaltung lässt sich dann begreifen als ein mehr oder weniger komplexes Akteur-Netzwerk, das die Verkettung von Operationen vollzieht und zugleich protokolliert.

Wer die Akteur-Netzwerk-Theorie für die Erforschung der Verwaltung nutzt, sieht sich unweigerlich mit der Notwendigkeit konfrontiert, Konzepte und Instrumente der Wissenssoziologie für die Auseinandersetzung mit einer Organisation zu nutzen, die gänzlich anders strukturiert und auch programmiert ist als Forschungseinrichtungen. Bruno Latour selbst hat diese Herausforderung angenommen und ein faszinierendes Buch über den französischen 'Conseil d'etat ' geschrieben, ${ }^{7}$ das gerade aufgrund seiner methodischen und konzeptuellen Außenseiterposition überzeugende Anregungen für die Politikund Verwaltungsforschung bringt. ${ }^{8}$ Fremdheit als heuristische Trumpfkarte - so wird der Hühnermann doch noch salonfähig im Hörsaal von Luhmann.

In unserem Band verfolgt Kerstin Brückweh eine derartige Strategie, indem sie nach der Wirkungsmächtigkeit einer Kategorie - des >Haushaltsvorstandes - für die administrativ-technische Erfassung der britischen Gesellschaft fragt. Die von ihr analysierte Statistik zählt ja spätestens seit der Jahrhundertwende zu einem Kernbereich der staatlichen Verwaltung, wie Jon Agar in seinem anregenden Buch zur >Government Machine` argumentiert. ${ }^{9}$ Für die Verwaltungsgeschichte bedeutet das die Herausforderung, die eigenen technischen Abläufe und Instrumente mit dem zeitgenössischen politischen Diskurs in Dialog zu bringen, wie es etwa auch Michel Foucaults Konzept des Dispositiv nahelegt. ${ }^{10}$ Ein solcher Schritt ist notwendig, aber dennoch selten, weil mit dieser Verbindung zwei unterschiedliche disziplinäre Welten miteinander in Beziehung gesetzt werden müssen. Hier taucht der Hühnermann plötzlich in der Schreibstube der Verwaltungshistorikerin auf.

Wer sich darauf einlässt, die Tätigkeit der Verwaltung in ihrem kulturellen und damit auch sprachlichen Umfeld zu verankern, wird schließlich auch die Verwaltungsakte selbst in einem neuen Licht betrachten. Sigrid Wadauer nutzt schon seit vielen Jahren ambitionierte textanalytische Verfahren, um signifikante Muster der Erfahrung und Aneignung von Wirklichkeit in autobiografischen Texten und nun auch in Verwaltungstexten zu erschließen. Dieses Zwiegespräch zwischen innovativen kulturwissenschaftlichen Verfahren und sozialgeschichtlichen Fragestellungen leistet einen wichtigen Beitrag zum Verständnis des Verwaltungshandelns, das sich eben nicht ausschließlich durch die Umsetzung normativer Vorgaben fassen lässt.

Eine andere Strategie in der Auseinandersetzung mit dem konzeptuell Fremden hat Peter Becker gewählt. Er nutzt konventionelle textanalytische Methoden im Blick auf die oberstgerichtlichen Entscheidungen in der Habsburgermonarchie während des Ersten Weltkriegs. Der Hühnermann als das Fremde ist dort in den Verfahren selbst präsent. Zahlreiche Beamte klagten vor dem Reichsgericht eine finanzielle Besserstellung ein - an der Stelle von loyalen, kaisertreuen und opferwilligen Dienern des Staates sieht man sich plötzlich mit den anderen, den selbstsüchtigen, an materiellen Vorteilen interessierten Staatsdienern konfrontiert. 
Wenn Luhmann mit dem Fremden in einen intellektuellen Dialog eintritt, steht auch die Position des Beamten als Funktionsträger in der öffentlichen Verwaltung zur Diskussion. Die systemtheoretische Eleganz, die den Beamten ausdifferenziert in eine Rolle und eine - zur Verwaltungsumwelt zählende Person, wird von Anthropologen und Soziologen eher als eine Einschränkung verstanden. In diesem Band nehmen wir die Herausforderung an, einzelne Disziplinen und Theorien im Blick auf Figur und Rolle des Beamten miteinander in einen Dialog zu bringen.

Caroline Dufour reflektiert als Expertin im Bereich der Verwaltungs- und Politikforschung die Aussagekraft von Pierre Bourdieus Theorie sozialer Felder für eine kritische Analyse staatlichen Handelns. Aus ihrer Sicht kann die Verwaltungsforscherin mit diesem theoretischen Instrumentarium eine perspektivische Distanz zum Objekt ihrer intellektuellen Begierde entwickeln. Bourdieus konzeptuelle Instrumente verfremden den Staat: Aus dem vertrauten Anzugträger wird der Hühnermann. Das methodische Potenzial dieser Analyse demonstriert Dufour an der Einführung des Leistungsprinzips in die kanadische Verwaltung. Ein politischer Patt und eine Koalitionsregierung der beiden großen Parteien besiegelte 1917 das Ende des alten Patronagesystems sowie die Einführung eines Systems, das mehr auf Ausbildung und Leistung als auf politische Zugehörigkeit Wert legte. Gleichsam als Nebeneffekt emanzipierte sich dabei das administrative Feld vom politischen.

Geschichte selbst kann zum Fremden im Eigenen werden, wenn die Verwaltungsforscher sich mit Transformationen von Staat und Verwaltung beschäftigen, um das bestehende System besser verstehen zu können. Frits van der Meer, Gerrit Dijkstra und Toon Kerkhoff beschäftigen sich dazu ebenfalls mit dem Leistungsprinzip im öffentlichen Dienst. Sie stellen sich in einen konzeptuellen Raum, in dem neben der vertrauten Figur des aktuellen öffentlichen Dienstes zwei fremde Gestalten auftreten. Diese beiden Hühnermänner stehen für historische Formen von Staatlichkeit mit einem ganz spezifischen Verhältnis von Aufgaben, Organisationsformen und Dienstethos. Die Autoren gelangen zum Schluss, dass die dezentrale Struktur des niederländischen Einheitsstaats die Flexibilität und Dauer erzeugte, um die unterschiedlichen Anpassungen an Aufgaben, Anforderungen und Dienstethos von Beamten im Nachtwächter-, Wohlfahrts- und aktivierenden Staat vorzunehmen.

Diese Themen werden von Valentin Seidler erneut aufgegriffen, jedoch aus einer postkolonialen Perspektive. Der Blick auf die öffentliche Verwaltung in afrikanischen Staaten aus der Sicht der Entwicklungsökonomie bietet einen fast schon privilegierten Zugang zur Reflexion über das Fremde im Eigenen. In seinem analytischen Raum ist jedoch die Ambiguität des Hühnermanns noch stärker ausgeprägt. Sein Interesse für die Verpflanzung von westlichen Ordnungssystemen in afrikanische und asiatische Staaten lässt vertraute Systeme wie unsere Straßenverkehrsordnung zum Fremden im neuen Kontext werden. Seidler nutzt nicht nur die Brille des Ökonomen, um diesen Prozess der Übertragung vertrauter normativer Ordnungen in neue politische und gesellschaftliche Situationen zu verfolgen. Indem er verwaltungsgeschichtliche und kulturwissenschaftliche Perspektiven als Ergänzung aufgreift, kann er 
den Hühnermann hinter dem Anzugträger erkennen. Das bringt spannende Anregungen gerade auch für die Entwicklungsökonomie.

Die Beiträge von Benjamin Schindler, Jean-Baptiste Fressoz und Burkhardt Wolf treten von einer jeweils dezidiert disziplinären Perspektive aus in einen Dialog mit der Verwaltungsgeschichte. Der Jurist Schindler setzt sich mit der Frage auseinander, welchen Stellenwert der Geschichtsschreibung in der Verwaltungsrechtswissenschaft zukommt. Dazu analysiert er zunächst die historischen Narrative in den Klassikern der Verwaltungsrechtslehre und fügt diese zu einer Art Metageschichte des Verwaltungsrechts zusammen. Dem paradoxen Befund, dass gerade die Geschichtsvergessenheit des Verwaltungsrechts seine Traditionsgebundenheit fördere, begegnet Schindler im zweiten Teil seiner Ausführungen mit juristischen Gründen für eine Geschichte von Verwaltung und Verwaltungsrecht.

Für den Wissenschaftshistoriker Fressoz stellen Verwaltung und Wissenschaft im 19. Jahrhundert Ressourcen füreinander bereit. Er zeigt am Beispiel der Pockenimpfung in Frankreich, wie sich um 1800 ein neues Verhältnis von Verwaltung, öffentlicher Gesundheit und der Konstruktion von medizinischen Fakten etablierte. Die enge Vernetzung dieser Bereiche und deren Auswirkungen auf epistemologische Praktiken eröffnen einen Blick auf eine Interdependenz, die man eigentlich als eine Hybridität bezeichnen könnte. Der Hühnermann als medizinischer Forscher mit amtlicher Zwangsgewalt tauchte in den Waisenhäusern auf und nutzte die Waisenkinder zur Produktion von Impfstoff. Komplementär dazu mobilisierten die Vertreter der Verwaltung die Bevölkerung zur Teilnahme an der freiwilligen Pockenimpfung, indem sie sich auf die Legitimation neuester medizinischer Forschung beriefen.

Burkhardt Wolf schließlich wirft einen literaturwissenschaftlichen Blick auf Mythen und Effekte der habsburgischen Bürokratie. Wolf unterzieht die in der Verwaltungsgeschichte einschlägig bekannten Erzählungen und unvollendeten Romane von Franz Kafka einer doppelten Lektüre, indem er diese zusammen mit Kafkas amtlichen Schriften liest, die er während seiner Tätigkeit für die halbstaatliche Arbeiter-Unfall-Versicherungs-Anstalt (AUVA) verfasst hat. Kafka erscheint dabei als das Fremde im eigenen Schaffen, als der Literat hinter den trockenen amtlichen Verlautbarungen und als der Bürokrat, der in den literarischen Bearbeitungen im Mittelpunkt steht. Dabei entwirft Wolf nicht nur en passant eine Poetologie der Verwaltung, sondern weist Kafka auch als einen - in nietzscheanischem Sinne - fröhlichen Bürokraten aus, der abschafft, wovon er schreibt.

Den Anfang der Klassiker-Relektüren macht Rüdiger von Korsigk mit einem Essay zu Thomas Ellweins zweibändiger Studie Der Staat als Zufall und als Notwendigkeit. Von Krosigk stellt die Argumente von Ellweins 1993 und 1997 erschienenem Opus magnum in den Kontext der gegenwärtigen Verwaltungsgeschichte und liest Ellweins Untersuchung als Dekonstruktion der rational und hierarchisch organisierten öffentlichen Verwaltung. Dabei entdeckt er im praxeologischen Konzept der »lebenden Verwaltung « eine Herausforderung und Inspiration für aktuelle verwaltungshistorische Forschungen. 
Die vorliegenden Beiträge treten nicht nur in Dialog mit der Verwaltungsgeschichte. Indem diese Zeitschrift Beiträge versammelt und so verschiedene Perspektiven (er-)öffnet, lässt sie auch verschiedene Disziplinen miteinander in Dialog treten. Insofern publiziert Administory nicht nur Diskurse über Verwaltung, sondern betreibt selber auch Diskursverwaltung, verkettet Diskurse und hält diese Verkettung von Operationen zugleich fest. Damit schreibt sich unser Jahrbuch in eine bestehende Diskursgeschichte ein, die mit der Emeritierung von Erk Volkmar Heyen und der damit verbundenen Einstellung des großartigen Jahrbuchs für Europäische Verwaltungsgeschichte 2008 eine zeitweilige Unterbrechung erfahren hat.

1 Erk Volkmar Heyen, »Entstehung und Arbeitsweise eines verwaltungshistorischen Periodikums: das गahrbuch für europäische Verwaltungsgeschichte`(JEV)«, in: administory. Zeitschrift für Verwaltungsgeschichte 1 (2016), S. 10-27, hier S. 14.

2 Ein Kunstwort, das die beiden englischen Begriffe administration und history zusammenzieht.

3 Ausstellung im kanadischen Pavillon während der 52. Biennale von Venedig, 10.6.-21.11.2007.

4 Cornelia Vismann, Akten. Medientechnik und Recht, Frankfurt a. M. 2000, S. 20; Jack Goody, The logic of writing and the organization of society, Cambridge 1986, Kap. 3.

5 Vismann, Akten, S. 22f.

6 Erhard Schüttpelz, »Was ist eine Akte?«, in: NCCR Mediality Newsletter Nr. 7 (2012), S. 3-11, hier S. 6.

7 Bruno Latour, La fabrique du droit. Une ethnographie du Conseil d'Etat, Paris 2002, in deutscher Übersetzung erschienen als Die Rechtsfabrik. Eine Ethnographie des Conseil d'Etat, Konstanz 2016.

8 Vgl. dazu auch die Beiträge in Marcus Twellmann (Hg.), Wissen, wie Recht ist. Bruno Latours empirische Philosophie einer Existenzweise, Konstanz 2016.

9 Jon Agar, The Government Machine. A Revolutionary History of the Computer, Cambridge, Mass. 2003.

10 Vgl. dazu die Beiträge in Andrea D. Bührmann, Werner Schneider (Hg.), Vom Diskurs zum Dispositiv. Eine Einführung in die Dispositivanalyse, Bielefeld 2008.

\section{About the Authors}

PETER BECKER is Professor of Austrian History at the university of Vienna. His research focuses on the history of state building and administrative reform in the Habsburg Empire and the First Republic and on the engagement of the Habsburg monarchy and its successor states with the internationalism of the late 19th and early 20th centuries. He has published widely on the history of public administration (cf. exemplarily Bürokratie, in: Docupedia-Zeitgeschichte, 30. 8. 2016, docupedia.de/zg/ Becker_buerokratie_v1_de_2016; Sprachvollzug im Amt. Kommunikation und Verwaltung im Europa des 19. und 20. Jahrhunderts. Bielefeld: Transcript 2011)

STEFA N N ELLEN leads the Historical Analysis Services of the Swiss Federal Archives that has a focus on history of administration. He holds a Ph.D. in History at the University of Basel. His research interests includes history of administration, cultural techniques, governmentality and history of madness and psychiatry. 